\title{
CHYLOUS ASCITES IN AN OPERATED CASE OF JEJUNAL GANGRENE - A CASE REPORT
}

Khalid Muqueem ${ }^{1}$, Gurunath H. Sutrave 2

${ }^{1}$ Associate Professor, Department of Surgery, Vijayanagara Institute of Medical Sciences, Bellary, Karnataka. 2Junior Resident, Department of Surgery, Vijayanagara Institute of Medical Sciences, Bellary, Karnataka.

\section{ABSTRACT}

\section{BACKGROUND}

Chylous ascites is a rare entity, which is encountered by the surgeon after surgeries like radical gastrectomy, duodenectomy, jejunal resection and retroperitoneal surgeries. It is the collection of lipid rich lymphatic fluid in the peritoneum due to leak of lymph from the intestinal lymphatics. The turbid or milky colour is due to its high triglyceride content.

\section{Case Presentation}

We present a case of Chylous ascites as a post-operative complication after resection anastomosis due to gangrene of a segment of proximal jejunum. Chylous ascites was managed conservatively. The output significantly decreased. This was followed by giving a diet of medium chain triglyceride oil. Patient eventually improved and discharged.

\section{CONCLUSION}

Chylous ascites can sometimes be encountered by surgeons after various abdominal surgeries. A detail knowledge of the causes and different ways of management is imperative, to equip a surgeon to deal with such cases.

\section{KEYWORDS}

Chylous Ascites, Jejunal Gangrene, Abdominal Surgeries, Post-Operative Complications.

HOW TO CITE THIS ARTICLE: Muqueem K, Sutrave GH. Chylous ascites in an operated case of jejunal gangrene - a case report. J. Evolution Med. Dent. Sci. 2017;6(18):1455-1457, DOI: 10.14260/Jemds/2017/317

\section{BACKGROUND}

Morton in 1694, first gave a detailed account of a 2-year-old boy who had accumulation of chylous fluid in the peritoneal cavity due to tuberculosis. Since then this condition of Chylous ascites is known to be a rare condition.(1)

Chylous ascites is attributed to malignancies as the cause in the majority of cases. Such cases where chylous ascites is due to malignancies, have poor outcomes due to obvious reasons. In case of chylous ascites in paediatric populations congenital lymphatic abnormalities may be the cause.

In our case, the cause was jejunal resection due to ischaemic gangrene of the jejunal segment. We conclude that inflammatory process due to ischaemic gangrene of jejunal segment could have initiated the process of damaging the lymphatic structure of the intestine. The following definitive surgery of resection and anastomosis of the segment may have caused physical damage or may have exacerbated the assault to the lymphatic structure of the proximal intestine, leading to the formation of chylous ascites.

We report here this unusual case of chylous ascites formation following resection anastomosis of gangrenous jejunal segment which was managed by parental nutrition, octreotide and supportive treatment. Our literature search did not yield results of report of any cases of chylous ascites following jejunal resection due to ischaemic gangrene of jejunal segment. Hence this report.

Financial or Other, Competing Interest: None.

Submission 21-01-2017, Peer Review 14-02-2017,

Acceptance 21-02-2017, Published 02-03-2017.

Corresponding Author:

Dr. Khalid Muqueem,

C/o. Department of Surgery,

Vijayanagara Institute of Medical Sciences,

Ballari-Cant, Ballari-583104, Karnataka.

E-mail: drshatkratu@gmail.com

DOI: $10.14260 /$ jemds $/ 2017 / 317$

\section{Case Report}

A 50-year-old woman was brought to our hospital with history of abdominal pain, vomiting and fever since 3 days. Pain was insidious in onset and progressive in nature. Pain became worse since last two days. Patient was vomiting bilious, non-projectile vomitous. She was brought to our centre as she was becoming increasingly sick and not responding to treatment by the local medical doctor.

On examination, she was febrile (102 F), tachycardia (100 beats/min) and blood pressure of about 90/60 millimetre of mercury (mmHg).

There was abdominal distension with guarding and rigidity all over. She was posted for laparotomy.

Intraoperative findings revealed gangrenous proximal jejunal segment of about $25 \mathrm{cms}$, the proximal extent of which was about $25 \mathrm{~cm}$ from the ligament of Treitz (Figure 1, Figure 2).

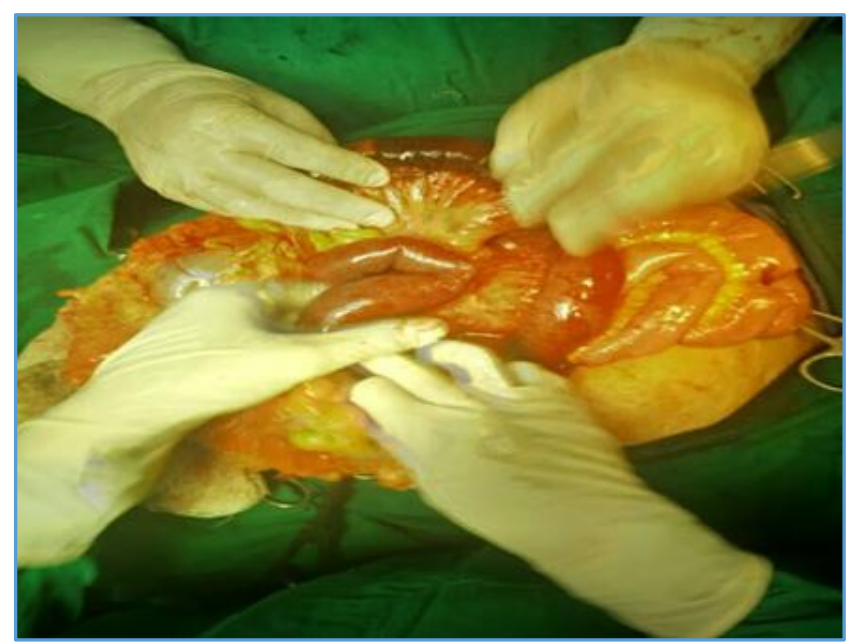

Figure 1. Gangrenous proximal bowel 


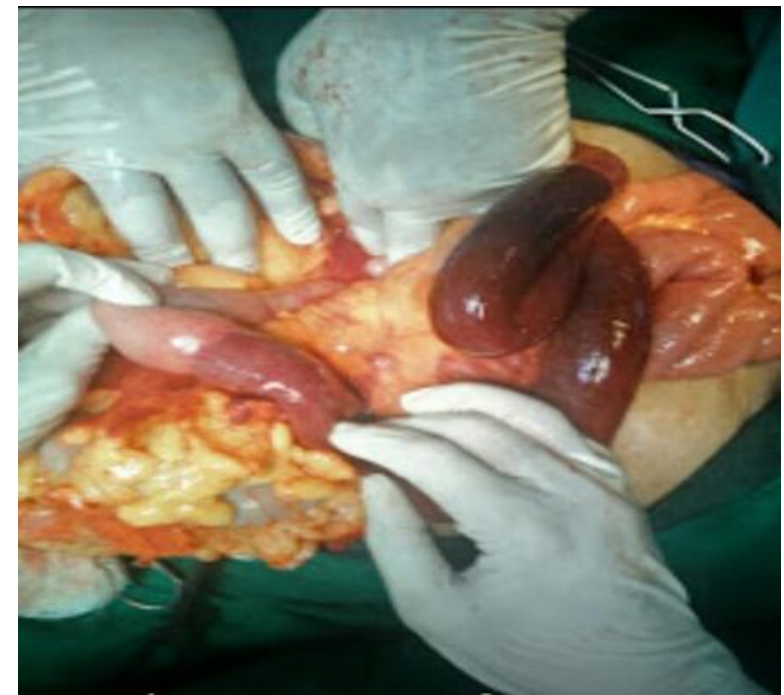

Figure 2. Junction of gangrenous and normal jejnum

The gangrenous segment was resected (Figure 3) and anastomosis of the healthy ends was done by double layer technique. Tube drains were kept near the anastomosis and abdominal wound was closed.

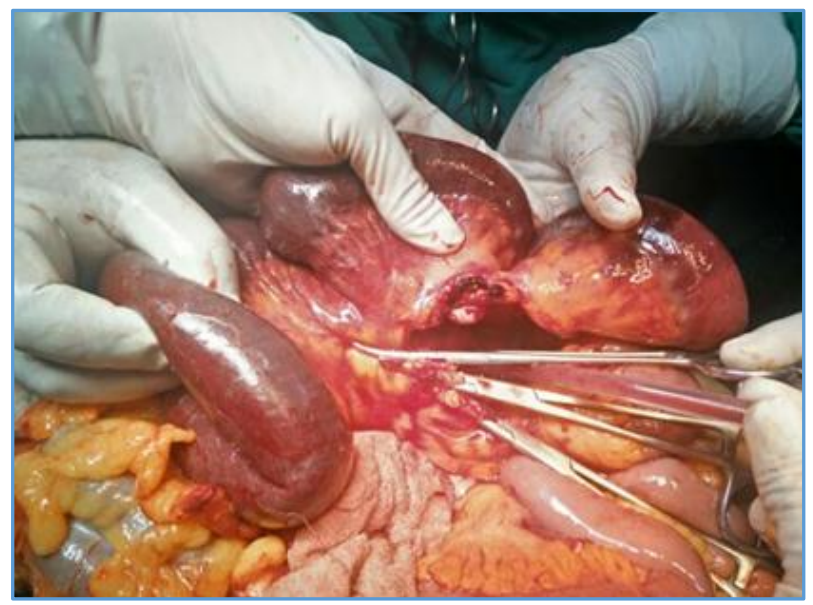

Figure 3. Gangrenous jejunum is being resected

Patient was extubated and recovered well postoperatively. Her haemodynamic parameters improved. But on the 2nd post-operative period, milky white ascitic fluid of about 1000 millilitre per day started coming from the drain (Figure 4, Figure 5). Analysis of the ascitic fluid revealed triglyceride levels to be $160 \mathrm{mg} / \mathrm{dL}$ and cholesterol level to be $16 \mathrm{mg} / \mathrm{dL}$. The aerobic culture of the fluid yielded no growth.

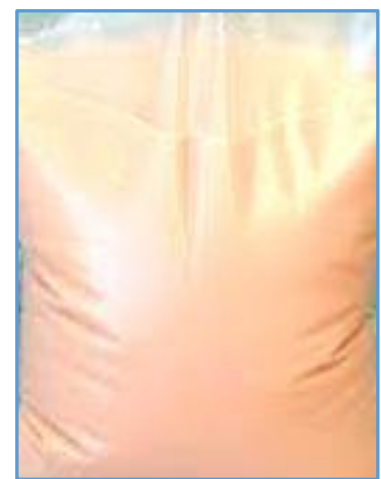

Figure 4. Milky chylous ascetic fluid collected in a bag
She was started on conservative management with octreotide and parenteral nutrition. Her drain output steadily decreased, reaching the reduced output of 100 - 150 millilitre per day on the seventh day. On day 7, octreotide was stopped and she was started on oral low-fat diet. During the second week, her drain output completely stopped. She was advised to continue this diet for the next 2 weeks.

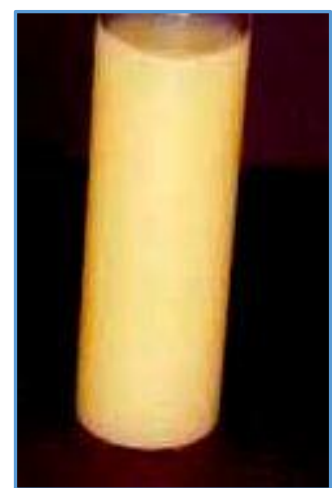

Figure 5. Chylous ascitic fluid sample for analysis

\section{DISCUSSION}

Chylous ascites is a form of ascites in which there is a high level of triglycerides in the collecting fluid. In large tertiary referral hospitals, the incidence of chylous ascites is reported to be in the range of 1 in 20,000 to 1 in 187,000 .(2)

To diagnose chylous ascites, at least one criterion among the following should be there: Lipid content of the peritoneal fluid is greater than that of the lipid content of the plasma; the ratio of protein content of the peritoneal fluid to the plasma protein content is > 0.5; the concentrate of triglyceride in the peritoneal fluid $>110 \mathrm{mg} / \mathrm{dL}$ and presence of microscopic fat.(3)

MR lymphography sometimes aid in the diagnosis of chylous ascites by visualising the major lymphatic leaks. But this is not helpful in case of small leaks.

Chylous ascites is formed due to various mechanisms such as - (a) Blockade of lymph vessels due to primary lymph node invasion by malignancy, (b) Lymph leakage from congenital megalymphatics in the retroperitoneum, (c) Mechanical trauma to the lymph vessels and/or thoracic duct during surgery or due to acute inflammatory process and (d) In congestive conditions like right-sided heart failure and cirrhosis of liver causing increased caval or hepatic pressure. ${ }^{(4)}$

Few cases are also reported of chylous ascites associated with intestinal obstruction. ${ }^{(5)}$

The above causes can be summarised as the traumatic causes and non-traumatic causes. Traumatic causes include those which occurs after surgeries (Abdominal or Oncologic) or after any therapeutic intervention. Because these type of interventions causes damage to the lymphatic vessels in the proximal mesentery and retroperitoneum.

Therefore, the incidence of postoperative chylous ascites in patients undergoing abdominal and retroperitoneal surgery is reported to be 1.1 to $7.4 \%{ }^{(6)}$

Radiation is also one of the causes of development of chylous ascites due to induction of fibrosis of the lymphatic vessels in the mesentery of small bowel, leading to obstruction followed by extravasation of chyle in the peritoneum.(7) 
In paediatric populations congenital lymphatic anomalies are the predominant cause of chylous ascites, especially in atraumatic cases. (8)

Chylous ascites is managed by employing different therapeutic modalities like low-fat diet with supplementation of medium chain triglycerides, octreotide, parenteral nutrition and in some cases even surgery is required.(7,9)

Octreotide helps in decreasing the chylous ascites by decreasing the splanchnic and portal blood flow. This causes decrease in portal pressure without causing significant effects upon the systemic circulation.(10)

In our case, patient showed good response with octreotide and parenteral nutrition. She showed steady improvement indicated by progressively decreasing output of chylous ascites.

Chylous ascites in a post-operative case of resection and anastomosis of jejunal gangrene is not reported in the medical literature to the best of our knowledge and after a meticulous online search. Hence, this case report.

\section{REFERENCES}

[1] Malagelada JR, Iber FL, Linscheer WG. Origin of fat in chylous ascites of patients with liver cirrhosis. Gastroenterology 1974;67(5):878-86.

[2] Almakdisi T, Massoud S, Makdisi G. Lymphomas and chylous ascites: review of the literature. Oncologist 2005;10(8):632-5.
[3] Cardenas A, Chopra S. Chylous ascites. Am J Gastroenterol. 2002;97(8):1896-900.

[4] Kaas R, Rustman LD, Zoetmulder FA. Chylous ascites after oncological abdominal surgery: incidence and treatment. Eur J Surg Oncol 2001;27(2):187-9.

[5] Steinemann DC, Dindo D, Clavien PA, et al. Atraumatic chylous ascites: systematic review on symptoms and causes. J Am Coll Surg 2011;212(5):899-905.

[6] Browse NL, Wilson NM, Russo F. Aetiology and treatment of chylous ascites. $\mathrm{Br} \mathrm{J}$ Surg 1992;79(11):1145-50.

[7] Aalami 00, Allen DB, Organ CH Jr. Chylous ascites: a collective review. Surgery 2000;128(5):761-78.

[8] Vignes S, Bellanger J. Primary intestinal lymphangiectasia (Waldmann's disease). Orphanet J Rare Dis 2008;3:5.

[9] Leibovitch I, Mor Y, Golomb J, et al. The diagnosis and management of postoperative chylous ascites. J Urol 2002;167(2 Pt 1):449-57.

[10] Leong RW, House AK, Jeffrey GP. Chylous ascites caused by portal vein thrombosis treated with octreotide. J Gastroenterol Hepatol 2003;18(10):12113. 